\title{
Enhancing the Appreciation of Traditional Chinese Painting Using Interactive Technology
}

\author{
Shichao Zhao ${ }^{1, *}$, David Kirk ${ }^{2}$, Simon Bowen ${ }^{1}$ and Peter Wright ${ }^{1}$ \\ 1 Open Lab, Newcastle University, Newcastle-upon-Tyne NE4 5TG, UK; simon.bowen@ncl.ac.uk (S.B.); \\ p.c.wright@ncl.ac.uk (P.W.) \\ 2 Department of Computer and Information Sciences, University of Northumbria at Newcastle, \\ Newcastle-upon-Tyne NE1 8ST, UK; david.kirk@northumbria.ac.uk \\ * Correspondence: s.zhao11@newcastle.ac.uk; Tel.: +44-783-538-4944
}

Received: 1 April 2018; Accepted: 13 April 2018; Published: 16 April 2018

\begin{abstract}
In this paper, we present a two-part study. The first part was a cultural appreciation study. Through this study, we explored the specific approach of cross-cultural aesthetic appreciation and mapped out the potential insights for a prototype design. In the second part, we carried out a design-led study. We designed a tablet application and conducted focus group studies to explore the interactive technology that assists in the support of cross-cultural audiences' aesthetic appreciation and engagement of traditional Chinese painting. Based on these findings, we went on to further explore an approach of interactive engagement which is specific to supporting cross-cultural appreciation, while also reflecting upon the interactive design suggestions for the development of aesthetic appreciation to offer various transferable insights to the Human-Computer Interaction (HCI) community.
\end{abstract}

Keywords: traditional Chinese painting; aesthetic appreciation; interaction design

\section{Introduction}

Although Chinese traditional culture is extremely diverse, Chinese ink painting has abundant cultural and philosophical history, which is known to be one of the most significant Chinese cultural heritages. Compared to other aspects of Chinese traditional culture, the performance and form of Chinese paintings is relatively commonly exhibited and appreciated. In an increasingly internationalised world, exposure to such a "foreign" art form is becoming increasingly common, and there is a broad range of interest in the maintenance of such artistic practices, which are otherwise dying out. For most amateurs, the appreciation of Chinese painting is an appropriate direction prior to learning the subject of Chinese painting. Aesthetic appreciation and engagement emphasises the unity and diversity of traditional arts [1]. The structure of the aesthetic engagement itself is found to be an intense involvement of attention in response to a visual stimulus [2]. Compared to the learning of drawing skills, aesthetic appreciation is a more appropriate approach to engage audiences to arts in their primary appreciation [3], but cultural diversity brings specific challenges to foreign audiences [2]. In other words, the features and structure of aesthetic appreciation provide a specific design approach, which could potentially support the experience of cross-cultural audiences.

On the other hand, interaction design offers extensive potential to help in the preservation of Chinese painting not only in China, but also on an international scale [4-6]. Researchers have used interactive technology in the representation of traditional cultural artefacts, which has often involved their digital augmentation, to support further engagement and appreciation. An important outcome of this is a broadening of their potential audiences, as well as the creation of new forms of cultural interactivity. Following from this radical design evolution, many interactive cultural projects are 
promoted as disseminating the objectives of traditional cultures, but an actual in-depth interpretation and engagement with the aesthetics of these cultural artefacts has received limited attention [7]. By carefully applying digital technologies, there is the possibility of enhancing cultural appreciation and information delivery. However, understanding how to best support cross-cultural appreciation and engagement with traditional Chinese painting is a specific topic that requires further exploration [8].

This study aimed to advance our understanding of the use of interactive technology to support cross-cultural appreciation of traditional Chinese painting. We used a mixture of questionnaires and in-depth interviews to support our initial data collection $[9,10]$. Simultaneously, we chose an Experience-Centred Design (ECD) [11] methodology, which enabled us to explore and capture data on the topic of cross-cultural engagement and aesthetic appreciation. In this paper, we report two correlative studies that explore the appreciation method of non-Chinese audiences, and then go on to describing the design of a series of fieldwork with non-Chinese participants, based on the mobile application we developed. Based on these findings, we further reflected on the interactive engagement approach, which is specific to supporting cross-cultural appreciation as well as reflect on interactive design suggestions for the development of aesthetic appreciation to offer various transferable insights to the HCI community. Before discussing the study, we first present existing work in HCI and its related fields, which informs and grounds our enquiry.

\section{Related Work}

\subsection{Digital Cultural Appreciation}

Previous research has shown how digital technology can be used to support the appreciation of painting in general. Most of these projects are displayed in museums or galleries. A prime example of this is an exhibition version of the Bian River scroll, which uses a touch-based navigation system as a form of interaction [12]. Likewise, there is a system that displays ancient Chinese paintings and displays the daily lives of the ancient people through technology, which can reconstruct images, audio annotations, and text labels. The digital inpainting of some images and information, which has been lost over time, is one of the main research areas for the system [13]. Specifically, in this research area, they used digital technology to represent an original painting or created an environment to support the appreciation of Chinese painting instead. In contrast, the aesthetic meaning of these paintings is disregarded and seems to be non-existent in this research approach. The implied meaning of Chinese painting is significant in supporting the audience's understanding, therefore emphasising the importance of aesthetical meaning to an audience is of great importance.

Some research projects focused more on the dissemination of cultural information. For example, there is a table-top system that presents an interactive viewing experience of Chinese painting, while also allowing users to gain information such as the dates and genres of the painting and artist visuals they are currently observing [14]. Another example was creating an app based on an iOS/Android system that allowed users to scan artwork for interpretive viewing purposes [15]. Giving audiences information through interactive technology is valuable, however, engaging them in appreciating the subtlety of paintings themselves is also an important potential element that is offered by interactive technology. While this previous research has been valuable, we supposed that digital appreciation should be based on introductory aesthetic knowledge and participatory expression, and not only specific to the displaying or interpreting of details and images of the painting itself. To summarise, we suggest taking an alternative approach on how to present an understanding of Chinese paintings, and how digital technology can inspire and expand the appreciation of Chinese painting from a global perspective [5]. Therefore, our intended goal was to present the elements and imagery of Chinese painting from an aesthetic perspective, using interactive technology to support greater audience appreciation of the aesthetic principles of Chinese painting. 


\subsection{Interactive Technology in Traditional Painting}

$\mathrm{HCI}$ has a long tradition of design and research for traditional cultural domains. This extends from digital preservation through exhibition aids, to the utilisation of technologies and interactive exhibitions. Some studies engaged specifically with how to preserve Chinese traditional culture, a notable example being Chinese calligraphy and painting, which today uses interactive technology, especially in the area of interactive creative drawing. For instance, some case studies developed interactive painting systems with a digital brush device that was based on a table-top environment or a mixed-reality environment [16-19]. In addition, some other projects created digital canvases with real paint brushes to conduct a real drawing experience [20,21]. Their findings highlighted that the interaction and simulation of ink-brushes offered a tangible drawing platform for users to experience line art in Chinese painting. However, a disadvantage of this is that the aesthetics of painting are difficult to communicate to users when using these kinds of platforms. For the exploration of novel artistic experience, some studies focused on multi-cultural interactive experiences. For instance, "Melodic Brush" created a cross-modal musical system that combines Chinese calligraphy and Chinese music to allow its users to experience a novel auditory representation [22]. There are several other objects that link drawing and music to use cross-modal mappings, therefore developing new art forms $[23,24]$. Many studies used camera detecting devices to capture brush footprints and water colour simulations, which are then able to output a painting within a table-top interface [25]. Novel interactive art installations (IAI) are another digital art system that had established a connection between novel interactions and Chinese paintings to construct an immersive artistic creation system [26]. Through the support of interactive technology, these projects created excellent opportunities to connect users' behaviours and thoughts to generate an output of paintings.

Nonetheless, most of these research projects focused on delivering experiences to users who have the same or similar cultural backgrounds, as well as for whom the aesthetics of painting may be common knowledge to. Thus, the main design goal is to support the skill experiences, such as interactive drawing. For this reason, we intend to explore a cross-cultural audience who is less likely to share aesthetic principles. Therefore, how to use interactive digital technology to support cross-cultural aesthetical appreciation will be the main research agenda for this project.

\section{Cultural Appreciation Study}

We investigated the aesthetic appreciation of Chinese traditional culture and Chinese painting based on cross-cultural interviewees, as well as the reasons and meanings behind the interviewees' understanding. Our specific research objectives were: (1) to explore the method of cross-cultural appreciation in traditional Chinese painting; and (2) based on our first research objective, to discuss the non-Chinese participants' understanding of colours in Chinese traditional painting and the meaning of Chinese painting elements. To address these objectives, we used questionnaires and in-depth interviews to explore the approach of appreciation, rather than exploring how digital technology itself can support Chinese painting. Through the findings in this section, we intend to inspire and outline the primary design elements of the tablet application prototype, which includes colours and relevant subjects, as well as an appreciation of emotional imagery, for the study design in the next section.

\subsection{Methodology}

As our main method, we chose an open-ended discussion and subjective interpretation from the aesthetic experience approach [2]. We utilised a workshop with participants who had different cultural backgrounds to analyse how they appreciate Chinese painting. In the first phase, we also compared the diversity of appreciation between both the Chinese and non-Chinese participants, which would reasonably support us to seek the specific appreciation method of the non-Chinese participants. In the second phase, we used questionnaires and semi-structured interviews to analyse the understanding of Chinese traditional colours and elements of Chinese painting. Through a series of 
activities and descriptions, we discussed the preferences of colours from the target audience to explore the understanding and symbolisation of colours and images themselves [27,28]. We use a mixture of qualitative and quantitative approaches, which would go further in refining the aesthetic appreciation approach focus on traditional Chinese painting and cross-cultural audiences $[9,10]$. This potentially transforms a range of design insights that support our study design. Combining the analyses from these workshop findings, we designed a prototype that could support cross-cultural appreciation of Chinese painting.

\subsection{Phase 1-Appreciation of Traditional Chinese Painting}

To seek the specific appreciation methods of audiences who have different cultural backgrounds, we referred to Beardsley's five criterion of aesthetic appreciation: object focus; felt freedom; detached affect; active discovery; and wholeness [1]. These five criterions were reflected in the seven questions which had been outlined by the three phases in the workshop. Table 1 shows each question that was discussed with participants. In this phase, we separated the non-Chinese participants and Chinese participants into different groups to explore the specific methods used in the appreciation of traditional Chinese painting. Before the workshops were carried out, we posted an announcement on a student forum which is based on a UK university's Students' Union and student society website, in which we explained the main process of these activities. Upon doing this, we began to search for participants who may be interested in traditional Chinese painting (this did not include any professional painters). We chose 12 participants with an age range of 20-40 years old, six Chinese and six non-Chinese; these participants were later divided into two separate groups, one group being Chinese participants and the other being non-Chinese participants. We prepared 80 samples of Chinese paintings, which included a plethora of types, times, and forms for each group to use as a source of material.

Table 1. The list of the main questions.

\begin{tabular}{|c|c|}
\hline Phase 1 & First Impressions \\
\hline Q1 & How do you begin to appreciate Chinese painting, and what is your starting point of appreciation? \\
\hline Q2 & What is the first point or element of the painting that you focused on during your appreciation? \\
\hline Phase 2 & During the Process \\
\hline Q1 & $\begin{array}{c}\text { Do you enjoy the process of appreciation, and was there any form of visual or sensorial discomfort } \\
\text { during your appreciation? }\end{array}$ \\
\hline Q2 & $\begin{array}{l}\text { Was there any experiences or understanding in the process of appreciation which you found hard } \\
\text { to describe? }\end{array}$ \\
\hline Q3 & Do you have any interesting findings that you would like to share with others? \\
\hline Phase 3 & After the Appreciation \\
\hline Q1 & Do you have any thoughts/any images you recollected after you finished the appreciation? \\
\hline Q2 & $\begin{array}{l}\text { Describe which method (steps/elements) you followed in your approach to appreciation and } \\
\text { explain how do these potential methods support your approach to appreciating Chinese painting? }\end{array}$ \\
\hline
\end{tabular}

Based on these five criterions, we divided seven questions into three phases: Phase 1: first impressions; Phase 2: during the process; and Phase 3 after the appreciation. Through browsing and attempting to appreciate each painting, participants were able to talk about each question with the other group members. This group discussion then went on to a further discussion, in which each participant wrote a few key words (representing an element) on sticky notes to share their own experiences and steps of appreciation with each other. In both groups, each participant was required to discuss and summarise the intercommunity from the key notes. This process of discussion formed group appreciation methods which were derived from non-Chinese participants and Chinese participants separately. Through this phase, we intended to compare and refine the 
aesthetic appreciation methods based on non-Chinese and Chinese audiences. In the next phase, we will specifically explore and expand on the appreciation method of non-Chinese participants.

\subsection{Phase 2-The Interpretation of Chinese Traditional Colours and Chinese Painting Subjects}

Based on the aesthetic appreciation method of non-Chinese participants we obtained in Phase 1, we specifically explored the subjective interpretation of visual elements (colours and subjects) of Chinese painting in this phase. Based on our main research target, which was about cross-cultural appreciation, all of our interviewees were non-Chinese. However, they did not attend Phase 1, and therefore were not influenced by our research results. We offered a colour atlas of 12 Chinese traditional colours for interviewees to choose from [29], as well as the following question: "Which colours/subjects do you think could represent Chinese painting and Chinese cultures and why?" After the questionnaire, we conducted semi-structured interviews with 10 interviewees to discuss the reasons and interpretations behind their choices. We set 50 questionnaires during an 18-month period-25 of them in Shanghai, China, and a further 25 in Newcastle, UK-with a total of 44 effective questionnaires in total. These locations were considered to find participants who varied in cultural background and ages. For the choice of interviewees, we considered several nationalities (German, British, Russian, Japanese, Korean and Spanish), as well as a varied age group (18-45). We did not require them to have any previous experience in drawing or appreciating Chinese painting.

The interpretation of colours in different cultures is much more complex and difficult than a study with the purpose of naming colours in different cultures [30]. We used a questionnaire to determine the main colours that are related mostly to Chinese traditional culture [31,32]. Furthermore, we also used semi-structured interviews as a primary method to explore a potentially deeper meaning behind the participants' choices of colours [33]. Based on the study by Yu on colours in traditional Chinese paintings, we used 12 traditional Chinese painting colours to gain an understanding of them, and also the imagery behind the colours themselves [29].

\subsection{Data Analysis}

Our collected data consisted of 44 effective questionnaires and six hours of audio data that was recorded during the workshops. We collated selective audio transcripts that formed colour demonstrations and elements of image boards. The gathered data were also supplemented by colour cards, participants' sketches, and the photos/images mentioned by participants. During the two cultural appreciation studies, we not only gained direct data that presented a distribution of Chinese traditional colours and elements of Chinese painting, but also intended to investigate the reasoning and interpretations behind that data specifically. More importantly, we had the opportunity to capture different voices of understanding from non-Chinese participants, which combined the data from different dimensions and perspectives to support cross-cultural appreciation.

\subsection{Findings}

Two distinct overarching themes were identified and analysed while covering the understanding of Chinese traditional colours and the subjects. In this stage, our findings and analyses focused on the appreciation methods of non-Chinese audiences (Colour-subject-interpretation), which is presented in Figure 1, and the effects of the understanding of Chinese painting based on the Chinese traditional colours and subjects: Figure 2 shows the frequency of occurrence of the colours mentioned in the questionnaire and the corresponding interpretations of the colour derived from each participant's choice. Followed by the structures in Phase 1, we further outlined the Chinese painting subjects and interpretations from the transcripts made by interviewees in Figure 3. For anonymity, we refer to participants as FP (participants from Phase 1) and SP (participants from Phase 2). 


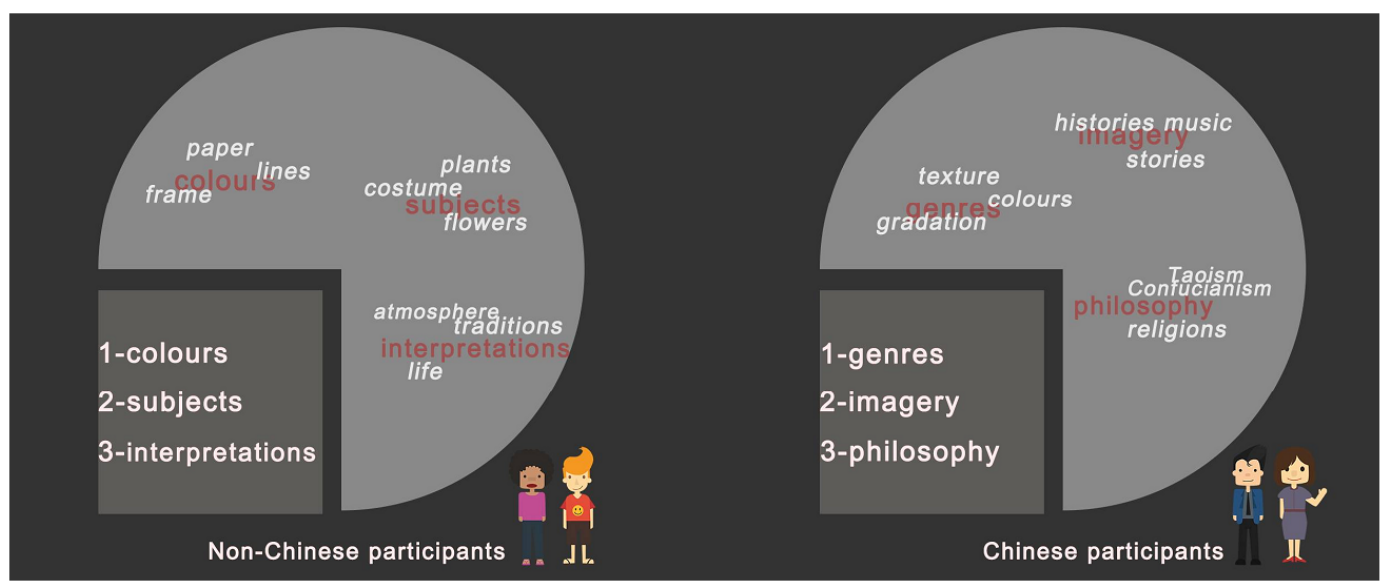

Figure 1. The structure pattern of appreciation methods.

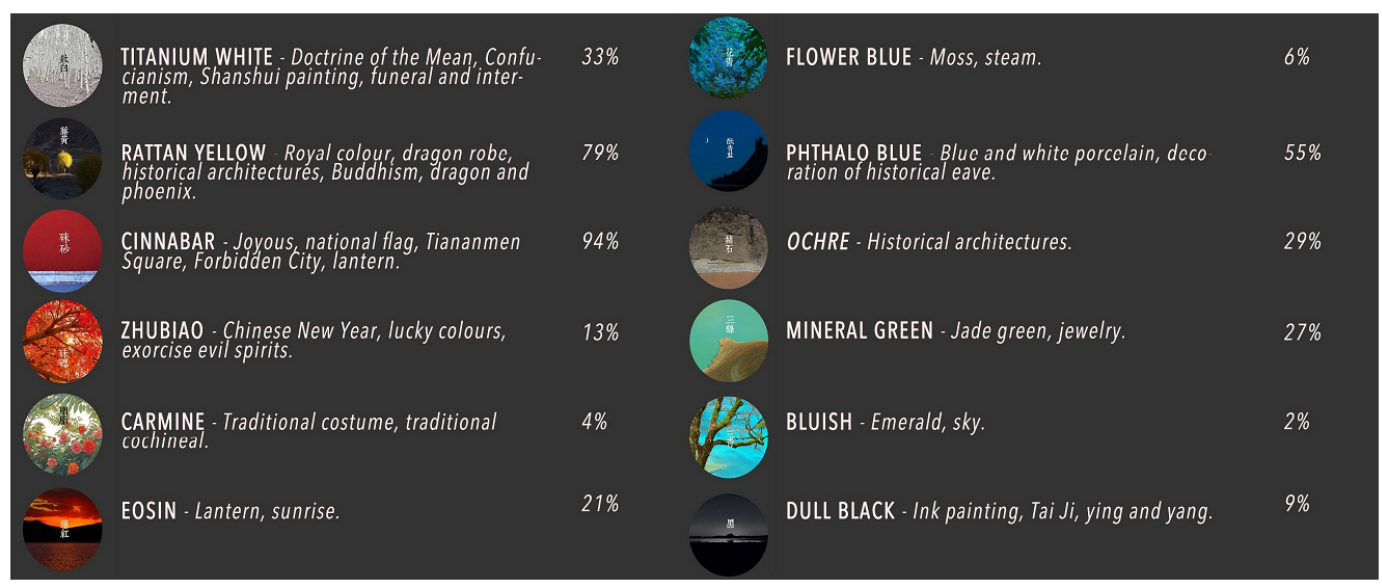

Figure 2. Understanding of 12 Chinese painting colours.

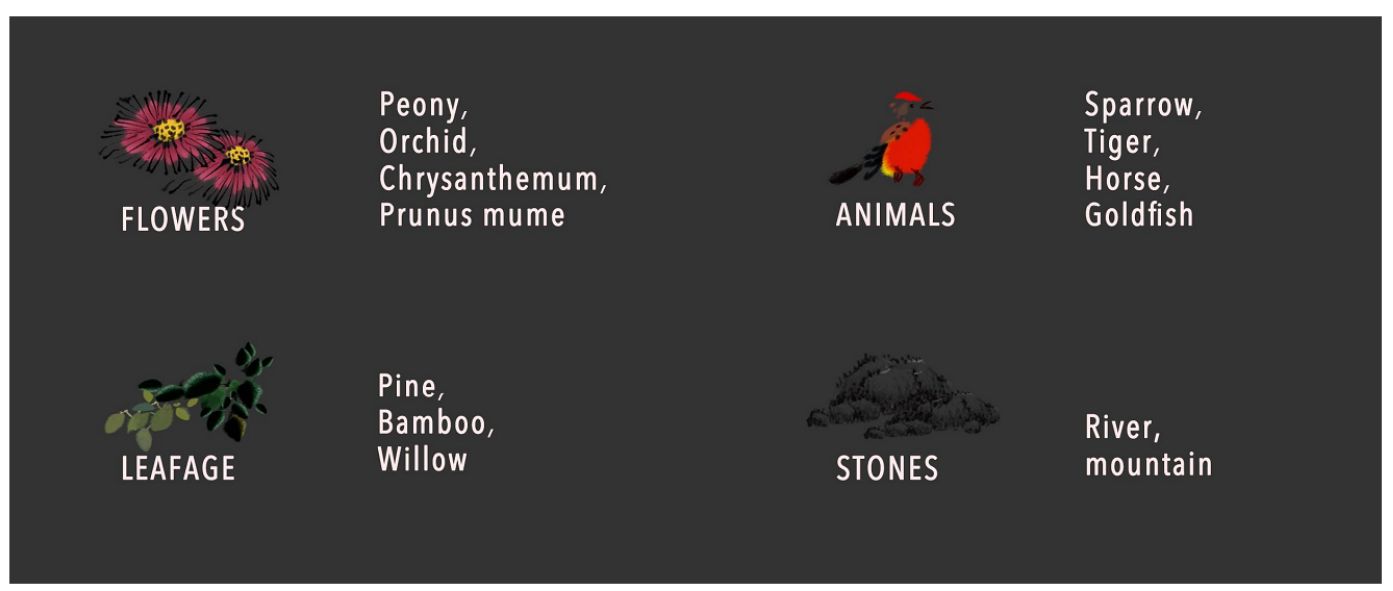

Figure 3. Interpretations of Chinese painting elements.

\subsubsection{The Method of Non-Chinese Audience's Appreciation}

From the analyses of Phase 1, we observed a notable difference in the way non-Chinese and Chinese participants discussed paintings. Figure 1 represents the main structures and elements of the two groups from their group presentations. For the first stage, the findings were focused on the primary impression of Chinese traditional painting. The colours of Chinese painting were the first 
element to be noticed by non-Chinese interviewees; the colours of the paintings were also seen as a basis to affirm the identity of Chinese painting. For instance, FP5:

"I think for me, it's really hard to describe Chinese paintings, so the colours are the first thing I managed to appreciate."

However, Chinese interviewees supposed that genres, such as elaborate-style painting or freehand brushwork painting, were the first element of their appreciation, rather than the colours of the painting itself. The second stage of the non-Chinese participants' appreciation was that the thinking of the subjects of the Chinese painting (based on the colours) were recognised by the majority of non-Chinese interviewees. FP2:

"We found that the subjects of Chinese paintings are very interesting, it's obvious that not just any random subject could be part of a Chinese painting. I think this is a good point as a basic starting point to appreciate Chinese painting."

Based on combining the understanding of each genre of painting, Chinese participants wanted to further get to know the expression of imagery and emotions in Chinese painting in the second stage. In the third stage, non-Chinese interviewees put an emphasis on the interpretations of Chinese painting, and the interpretations were built on an understanding of colours and subjects. FP6:

"I think this painting is trying to create a peaceful atmosphere here, from the green mountains, rivulet, to the village...I can feel the interpretations of the subject from these."

However, the degree of interpretation depended on an understanding of colours, subjects, and other elements. FP1:

"I won't say I am guessing the meaning of this painting, because I try to find the meaning from the colours, the bamboo and also the orchid."

This was different from the Chinese interviewees, whose third stage was more heavily focused on the experience of the philosophical meaning, which was combined with their personal understanding from the first and second stages.

\subsubsection{Complementary Understanding of Colours and Subjects}

We analysed the participants' understanding of 12 traditional Chinese colours from Phase 2, as well as their interpretations of the subjects in Chinese painting. The frequency rate of the six colours (which had been mentioned by participants) was less than $25 \%$ and the rate of three colours (which had been mentioned by participants) was less than $10 \%$. More than half of the Chinese traditional colours were not accurately appreciated by the participants. Based on these data, we found that incomprehension of traditional colours caused a deviation in the appreciation of Chinese painting to a certain extent. SP3:

"Actually, I just thought that red, yellow, black and white were Chinese painting colours. More than half of these colours I have never known, so I can't say or describe the meaning of them."

However, the meaning behind these traditional colours did develop interest, SP1:

"Is it a kind of mineral pigment? So, what is it normally used to do?"

Based on the understanding of colours, the subjects of traditional Chinese painting played a complementary role in the appreciation of Chinese painting. We classified four different types of subjects in Figure 3 which were mentioned and summarised, corresponding to the interpretations of the participants. Meanwhile, the subjects were accepted by non-Chinese interviewees as entry points to learn how to appreciate Chinese traditional painting. SP8: 
"The subjects of Chinese painting are really helping me to understand what the painters want to actually express, and also it helps me to get to know the Chinese traditional cultures."

The subjects of Chinese painting also cover the incomprehension of colours, SP7:

"I can't say that I am guessing this, I am trying to use my understanding of these animals and plants to conjecture the meaning, even like what I said before, I don't know the meaning of this specific colour."

SP1:

"If I browse more paintings, then I can easily classify paintings based on the subjects shown."

\subsection{Critical Reflections}

Conducting the workshops and interviews with participants who have a multi-cultural background yielded a productive design framework. (1) Appreciating method: Using a visual stimulation of colours associated with Chinese painting subjects to transform a series of aesthetic interpretations is an acceptable method for non-Chinese participants; (2) Design potentials: Some incomprehension of Chinese painting colours and subjects still exists within the appreciation. Elemental archive of colours and subjects may offer a potential method to supplement a reasonable understanding; (3) Colour-subject-interpretation: These three points are reversible and complement each other in the process of appreciation. During the appreciation, the process of one element transforming another element would potentially create the interaction and engagement between an audience and Chinese paintings. Aesthetic experience is not only limited by visual viewing, but also by interactive expression. These design reflections will be further embodied in the design study.

\section{Design Study}

Following the two initial activities' findings (questionnaires and in-depth interviews), we now describe our study design. Our research questions were: (1) How do cross-cultural audiences appreciate and engage with traditional Chinese painting based on tablet device use? (2) How is interactive technology specifically impacting the aesthetic appreciation for cross-cultural audiences? To address these questions, we designed a prototype application, intended for use on a tablet-style device and conducted workshops using the prototype to explore further design challenges.

\subsection{Methodology}

We utilised the experience prototypes and interviews of focus groups from the point of view of the Experience-Centred Design (ECD) [11] methodology, which enabled us to explore and capture data on cross-cultural aesthetic appreciation and engagement. Based on the analyses and findings of the previous Cultural Appreciation Study, we designed content suitable for an iPad app. This app not only expressed and developed the findings that were gained from the cultural appreciation study, but also integrated interactive technology to explore design insights and challenges that were specifically aimed at supporting cross-cultural audiences to appreciate traditional paintings. We explored this app with participants from diverse cultural backgrounds, in a series of user tests and interviews, to engage them in experiencing the app and sharing stories in their artworks. Participation in the study involved: An introductory session run by the researchers to explore different methods of engaging with traditional painting, then a tutorial on how to use the app, and then a group interview to compare and contrast different methods of engagement. This was followed, for a subset of participants, with a further in-depth group interview to discuss the features of the design and its interface.

\subsection{Recruitment}

We recruited 10 participants (from four different countries) to test and experience the app, ranging in age from 8 to 46 years. The participants were allocated into four groups: (1) a British family of three 
(two parents and an eight-year-old child); (2) three British/Spanish students in university; (3) two Korean/Chinese staff who worked in a gallery; and (4) a British middle-aged couple. All participants described themselves as highly interested in traditional Chinese painting, and three participants stated that they used to engage Chinese painting or calligraphy with an ink-brush, prior to this workshop. We hoped to analyse diverse users who have a different cultural background, age group, and proficiencies, which would therefore provide valuable critical accounts to use.

\subsection{Prototype Design}

In the previous section, we summarise the findings that combined the specific method of non-Chinese participants' appreciation and their understanding of colours and subjects in traditional Chinese painting, which inspired us to design the primary prototype. Based on the previous Cultural Appreciation Study, we set our design sights on the cross-cultural appreciation approach (colour-subject-interpretations) with elemental expression to engage participants in appreciating and experiencing traditional Chinese painting. This highlighted the specific appreciation method for non-Chinese participants that explored their technique of understanding and needs of engagements. Since more than half of our participants were iOS users, and considering the operability of gestures, we selected and designed a tablet application based on an iOS system as a prototype. A key design feature was that we used elemental painting subjects with tangible operations to support participants who had a varied cultural background to appreciate and engage with traditional Chinese painting. Accordingly, we used participants with diverse cultural backgrounds, ages, and degrees of proficiency with traditional Chinese painting. To address our research questions, our tablet-based application needed: (1) to be an elemental archive that provided multiple colours, subjects, and styles of Chinese painting that could support various dimensions of appreciation; (2) to offer diverse elements from the archive to users to encourage the creation of their own artwork; and (3) to be a platform that engaged users to make collaborative artwork.

The iOS app was installed on an iPad, and was given to each participant group for exploration. The app included an interface with various types of Chinese painting elements, alongside PNG images that had no background (transparent). These were intended to be used to develop a piece of artwork. We referenced the relative Chinese painting drawing skills [34] to reconstruct all of the elemental images to vector images, rather than bitmap scans, of the original paintings to ensure that the resolution and integrity of the elements were of a high quality (Figure 4). There were 40 pieces of imagery in the elements of the library, and three different functions were used in the main interface (Figure 5a). The main interface could be used as a canvas for participants. In this interface, we displayed one icon in the right corner; thus, upon use, displayed a further three different icons consisting of: adding another element, removing an element and saving a current canvas as an image (Figure $5 b$ ).

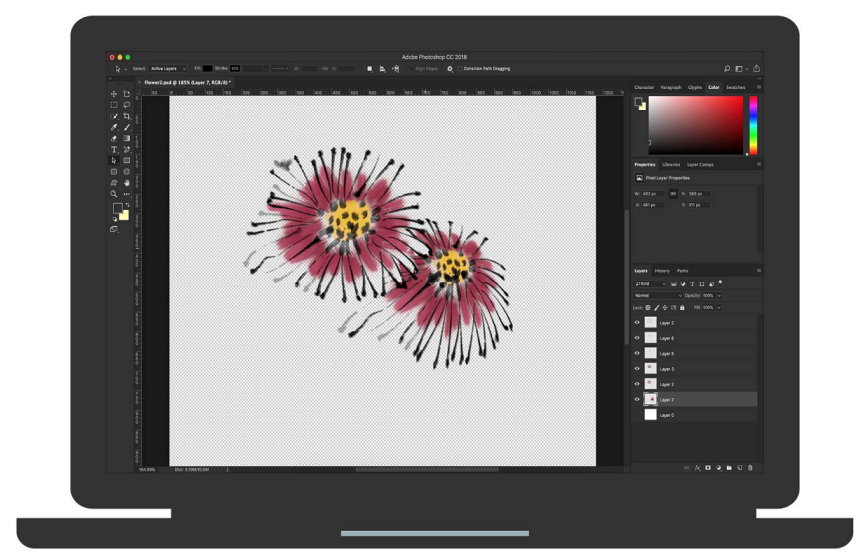

Figure 4. Interpretations of Chinese painting elements. 


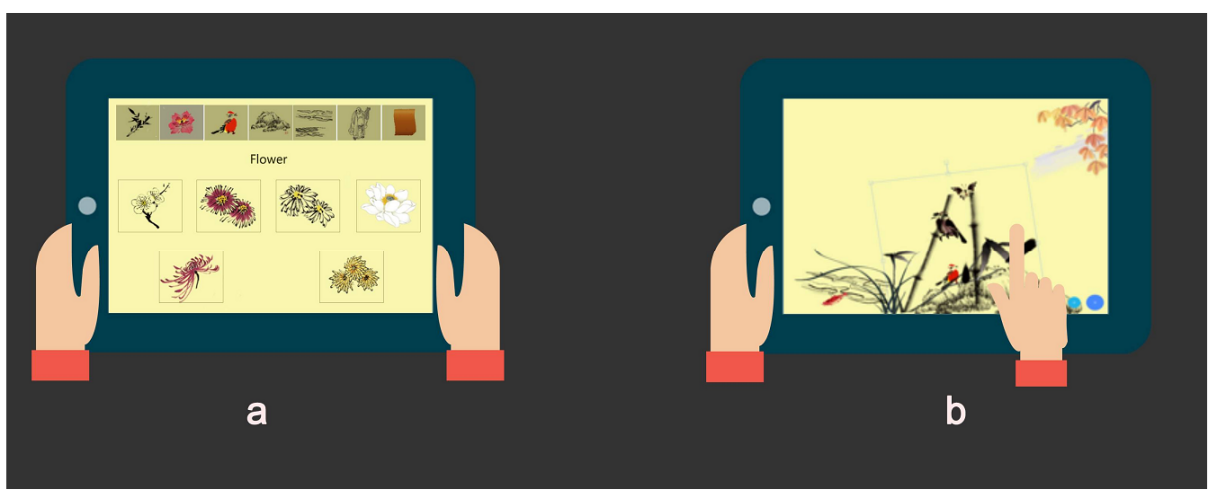

Figure 5. (a,b) Interpretations of Chinese painting elements.

When adding elements, any elemental image can be chosen and then shown in the canvas; the methods for doing this were single choice or multiple choice. In the multiple-choice option, different elements are shown on the canvas, but have their own individual layer, depending on which element was added first. This layered concept is convenient for participants to collaborate with each other and change elements with ease. When removing elements, participants would be able to remove any element in the canvas individually just by simply selecting it. For any element that the user wanted to choose, they were able to use a multi-touch function to adjust the position, size, and proportion, as well as being able to use their fingers to allow for each element to be mixed with another element interpretively, forming a piece of digital artwork. Meanwhile, all of the elements had a transparent background to offer participants further possibilities when attempting to accomplish complicated and multi-layered artwork. After participants finished a piece of artwork, they needed to use the save icon to save a current canvas, which was then saved as a JPEG on the device. Upon saving, participants were still able to add new elements to the canvas. The format of the photos were JPEG images, which allowed participants to print it or to quickly post it online.

\subsection{Procedure}

We arranged the 10 participants into four groups (see above) for an introductory session. In the session, we provided participants with two different ways of engaging with Chinese painting: Firstly, a Chinese painting website (http://www.ukctc.org) which included English translations and descriptions of various genres and dynasties. Participants were also provided with ink brushes, pigment for Chinese painting, Chinese art paper, and ink stones, to support participants in appreciating the traditional approaches to Chinese painting. Participants were given $15 \mathrm{~min}$ to explore the materials.

Secondly, participants, in their groups, were then given an iPad with the application pre-loaded. They then received an explanation for using the application-including the concept of the elemental archives-and given an opportunity to ask questions about the app. Participants were then allowed to explore the app together, which they did for an average of $30 \mathrm{~min}$. After each group finished exploring the two forms of experience, the participants were invited to group interviews to describe their experiences and discuss the advantages and disadvantages of these two forms of engagement. The questions focused on the differences and preferences of these two formats with regards to engaging with traditional painting. It is important to stress that we chose different deployment locations, which included a family living room, a gallery, and a studio, which supported us in exploring appropriate settings for appreciation.

We held a further user-experience interview following the four group sessions as a secondary activity. We invited one participant from each group to take part in an in-depth interview. Participants engaged in a discussion on the topic of their user experience using the application. The interview schedule was semi-structured, and many of the questions were oriented towards user experience, but others prompted a conceptual discussion of the effects of interactive technology in the cross-cultural 
appreciation of traditional art. This discussion incorporated how to engage participants in experiencing Chinese painting, and also explored their self-expression within artwork and how this impacted their engagement with, and experience of, traditional painting. Critical reflection on the design and usability was also discussed, which supported us in iterating the design of the application. Table 2 shows each question that was asked and discussed with interviewees in Phase 1 and Phase 2. All interviews were audio-recorded with the participants consent.

Table 2. The questions of the two phases.

\begin{tabular}{cl}
\hline Phase 1 & \multicolumn{1}{c}{ Group Engagements } \\
\hline Q1 & Do you think these two methods of experience are different for you and why? \\
Q2 & Which method do you prefer and why? \\
Q3 & Can you talk about your paintings/artworks? \\
Q4 & Do you have any issues/problems when you are doing this? \\
Q5 & Which painting are you most satisfied with and why? \\
Q6 & Do you want to share your artworks or talk about it with other people? \\
Q7 & What have you achieved through these two activities? \\
\hline Phase 2 & $\quad$ User-Experience Interview \\
\hline Q1 & Do you understand the main function and purpose of this application? \\
Q2 & Do you think that this application is helpful in your understanding of Chinese painting? \\
Q3 & If you think this application is useful, which part of your experience was your favourite? \\
Q4 & If we let you be the designer, are there any suggestions you have for us to add into the application? \\
Q5 & (i.e., new interactions or operations) \\
\hline
\end{tabular}

\subsection{Data Analysis}

The data were analysed through the process of Thematic Analysis [35]. Initial codes were generated and refined using iterative analysis to produce coherent themes that were then further refined to establish useful findings that contributed to the exploration of cross-cultural appreciation of traditional Chinese painting, using interactive technologies. The data that were collected consisted of audio data recorded during interviews, paper prototypes and design sketches (provided by participants). From the two interviews, we classified data as GA (data from group engagements with the application) and UX (data from the use-experience interview). Below, we discuss the three main themes that had emerged from these data, which are: (1) Preference and Barriers in the Appreciation; (2) Self-Expressions in the Appreciation of Artworks; and (3) Collaborative Engagements.

\subsection{Findings}

In the following sections, we explore in further detail some of the key features of our participants' experiences while using the prototype application, as well as exploring their reflections on using interactive technology to support cross-cultural appreciation of traditional Chinese painting. The findings reflected on three areas of interest; firstly, we discussed the experience and feedback of our cross-cultural appreciation approach to Chinese painting. Secondly, based on the responses and practice of using our application, we explored the participants' self-expression in the appreciation of Chinese painting itself. Thirdly, we reflected on some of the participants' thoughts on mobile-access artistic appreciation, which focused greatly on the gestural interaction of mobile technology and collaborative artwork.

\subsubsection{Preference and Barriers in the Appreciation}

Based on a comparison of two different engagement approaches, the participants often discussed the barriers that they met during their appreciation process. One of these barriers included how to seek a starting point for appreciating something. During the activity, the images that had descriptions in the traditional appreciation approach did not offer an appropriate starting point for non-Chinese participants. However, the application offered GA3 another method to appreciate Chinese painting, 
which supported them in accordance with their usual method of understanding. Painting subjects through elements can appear to be specifically detailed through the use of colours, while also providing a reasonable starting point that could support non-Chinese participants' understanding of Chinese painting; as GA3 explained:

"Actually, I was confused, because I don't know much about Chinese painting, so these websites gave me some rigid knowledge on the topic of it. To be honest, I don't even know where I should start... so I just started with some very random browsing. But I do quite like this application, I gather that I should start from these elements, even though they didn't show me a direct description of Chinese painting. I feel as though the elements of these subjects are presenting the meaning. I think it is useful for non-Chinese audiences as I think we do need these elements to have basic cultural understanding if there is nothing else to build this understanding on."

Some of the participants believed that cross-cultural appreciation needed to have a previous step of understanding as a method of support. The separate elements interpreted the potential meaning of a painting, but also preserved imagination for the appreciation of the paintings as a whole. In GA10's words:

"I think this is very different with the normal way we approached appreciation beforehand. Because using elements as an explanation point could help us understand the painting much more easier. For example, it's telling us this flower is from a Chinese painting, and this is what it looks like in the Chinese painting per se. But the elements did not limit our own imagination and understanding, the elements are supporting our understanding rather than telling us how to appreciate the painting itself."

In the discussion of the relationship between elements and traditional Chinese painting, the participants collectively voiced that, as a method of approach, the subject elements also offered a progressive appreciation that extended the understanding of Chinese painting to Chinese traditional cultures. As GA7 elaborated:

"I enjoyed getting to know Chinese painting through this route, while showing me some parts of Chinese painting, it also introduced me to thinking about the special meaning behind the elements themselves, for example plum, orchid, bamboo and chrysanthemum. This informs me of what the interpretations of these are in Chinese culture. Now, my appreciation is not only limited by visual senses, but through many other methods too."

Multiple dimensions of elements were also mentioned numerous times by the participants. Based on the subject elements that could potentially support the interpretation of Chinese painting, some participants suggested that adding textures and composition as a feature into each element would be helpful in supporting appreciation of Chinese painting on a greater scale. For example, GA5 explained:

"Obviously, adding more subjects in this archive would support us to understand a much more in-depth meaning that sits behind this painting. But in all honesty, I really want to know the composition of Chinese painting, which is very difficult to grasp from a canvas. Nonetheless, I hope the archive can add something like this as it would help support not only mine but a lot of other people's appreciation of Chinese painting."

\subsubsection{Self-Expression in the Appreciation of Artworks}

Being a tablet-based application, we designed a framed platform that would support the combination of interactive elements derived from digital archives, allowing for the expression of participants' understanding. Based on our research question, this framing prompted further interesting reflections from participants. The participants' expectations of appreciation were limited to browsing paintings or learning the skills, in which UX4 supposed that the self-expression of painting appreciation could also be used as a reasonable approach to understand the interpretation of traditional Chinese painting: 
"I think it's good to combine these elements in order to express myself, I can put my own stories into this. But this does ask me to have a satisfactory understanding of Chinese painting. However, when experiencing this, I am also having an inner drive of artistic knowledge."

Compared to the first approach, in which the participants experienced Chinese painting with an ink brush, which emphasised on the experience of drawing, participants were clearly interested in performing self-expression with the elements from these archives instead. However, while the application we offered weakened painting skills, it did, in fact, strengthen operability. GA9 gave her feedback on self-expression and learning skills:

"The first method leans more towards the learning of Chinese painting rather than appreciating it. I prefer the self-expression method based on the appreciation and not the drawing. Just like when I go to a gallery to talk about a painting, it doesn't mean I want to learn how to actually do it."

Meanwhile, self-expression was closely connected to the participants' interest in Chinese painting. Lack of painting skills resulted in participants easily losing interest in painting, discouraging their entire process of self-expression. UX1 shared her thoughts:

"As a foreigner, it's so hard to let me express something with an ink brush, I can't even draw a straight line here... I think this is the reason behind me giving up so easily."

During their experience of the application, the method of elemental combination ensured the integrity of artwork, as well as providing feasible operation with an ink brush. As UX3 elaborated:

"This way is more of a specific design approach for doing self-expression during appreciating something, maybe this is because it closes the distance between us and Chinese painting. The painting is not out of reach anymore, we can share our emotions and stories within it which is just a fantastic experience."

In the discussion with participants, we discussed self-expression in appreciation, and also distinguished the difference between skill learning and self-expression in the method of appreciation. This supported us in ensuring that our design direction was correct when it came to the appreciation of Chinese painting.

\subsubsection{Collaborative Engagements}

A lot of feedback was heavily focused on the collaborative engagement with Chinese traditional painting. Through discussion, participants stated they could use the application we offered to form a collaborative piece of artwork. Connected to this, interactive technology was specifically discussed in the in-depth interviews. The application we designed used multi-touch technology to support participatory collaboration. Collaborative artwork could be used as an appreciation approach for the participants, and, more specifically, as a method to improve their participation in the process of appreciation. Based on this, the interaction was not only between participants and paintings, but also expanded the interaction itself, between participants.

For GA6, the collaborative engagement potentially offered a form of communication between him and his child, rather than a sort of educational guidance regarding painting appreciation:

"You know it's really hard to teach him how to appreciate painting... if I just read some books or showed him some paintings, he might just get distracted and go away. But this is fascinating, we can talk about this, we can even touch it and check the details of it."

Meanwhile, from the in-depth interviews, we found that collaborative art work could be seen as an "adhesive" to connect more details of the elemental archive and appreciation. The discussion from participants had more specific content rather than abstract content, as personal stories and emotions were expressed in their discussions as well: UX2: 
"I think when we draw together, it's more like a form of artistic communication. Through this, we can talk about composure, colours and also the meaning of the painting. It's not like a professional discussion, but so many details are covered, in honesty, it's quite emotional."

However, some participants reflected their expectations of the process of collaborative engagements. Graphical representations or interactive notes would be a helpful tool in providing more aesthetic interpretations, for example GA7 mentioned:

"If we could have more graphical suggestions to present us on how to experience this particular application, it would be more affective. What I mean is, different participants get different graphical targets in the engagements, therefore through discussion we could talk about it."

More technical needs were also shown in the discussions, such as multi-touch and gestural adjustments of the elements that would give participants additional interactive possibilities. However, most importantly, the connection with social media was also discussed; UX1:

"I would like to share this and post it on my social media, because if I do, it might encourage more engagement and interest in this application, expanding the discussion and making collaborative artwork more meaningful and appreciated on a greater scale."

\section{Discussion}

Based on the findings from the cultural appreciation study and the design study, we now turn to elaborate our contributions of this study to the digital cultural heritage community: (i) a set of findings that explored a specific engagement approach for the cross-cultural appreciation of traditional Chinese painting; and (ii) a series of interactive design suggestions and challenges for the development of aesthetic appreciation in Chinese painting. Our aim was to not only utilise the application to represent our findings in the cultural appreciation study, but also reviewing the design suggestions and reflections from the evaluation. Our proposed contribution is constructed on the qualitative and quantitative analyses, which we were able to generate, as well as the values and concerns that had been communicated through the experiential reflections on the application which we designed.

\subsection{Element Archive_Engagement Approach for Cross-Cultural Appreciation}

A key area of concern for cross-cultural appreciation is the following of the appreciating method and understanding of the approach of an audience who has a different cultural background. The understanding of colours is an appropriate starting point to guide a non-Chinese audience to appreciate Chinese painting. As a starting point, colours could potentially connect to the cultural meaning of the subjects within the painting, which would then interpret the aesthetic appreciation to the audience and inspire them to understand the traditional cultures through interpretation [36,37]. However, how to combine and connect these elements as an engagement approach is what we focused on in the following sections.

We performed an in-depth analysis based on the understanding of the elements in Chinese paintings from participants, thus informing us that, in general, colourific subjects were greatly accepted by participants. From the interviews, we could see that the colours that were chosen were described by figurative subjects and personal experiences. By grouping several colours together, this offered the audience images and impressions of Chinese traditional culture, architecture and other figurative subjects that could also be used as a method to support the Chinese and non-Chinese audiences' understanding of culture. Conceptualising colours to subjects, and concreting subjects to colours are iterative processes, and only using colours to understand Chinese traditional painting is unreasonable for non-Chinese audiences. However, combining the understanding of traditional painting colours with the expression of subjects could potentially map out a method of appreciation [38]. Collectively, participants argued that the elements archive could be an approach to combining different elements (i.e., colours, subjects, etc.) to support cross-cultural appreciation. For the non-Chinese audiences, 
holistic aesthetic appreciation was not that easily accepted. The elements archive gives more of a flexible method to guide audiences in exploring the different dimensions of Chinese painting by themselves, as well as not limiting their own understanding and imagination.

Simultaneously, based on participants' experience and engagement of the application we designed, participants complemented more dimensions that were for supporting the elemental archives. Colour-subject-interpretation as an appreciating method showed just one approach to appreciation, but was not limited to only this approach. Therefore, combining our findings, we classified these three elements (colour, composition, and texture) for the archive. First, the interpretation of Chinese traditional colours was diversified, and the origin of Chinese traditional colours differed from the interpretation of Western contemporary colours. Therefore, the integrated and visual interpretation of Chinese colours could provide an opportunity to support the appreciation of Chinese painting. Secondly, extraction of the composition of Chinese painting has its own theory, which is similar to the perspective effect and inverse perspective effect [35]. Understanding the composition of Chinese painting would support its appreciation dramatically. Thirdly, the understanding of textures in Chinese painting offers an audience an in-depth method of appreciation [35]. Overall, the understanding of the subjects may be strengthened and may become more concrete due to these elements. Therefore, the subjects could be seen and used as a further elemental source that interprets the audiences' understanding of the subtle aesthetics in Chinese painting (Figure 6).

In conclusion, using the elemental archive as an engagement approach could support non-Chinese audiences' aesthetic appreciation in the area of traditional Chinese painting. This is potentially a very rich area of cross-cultural appreciation in art.

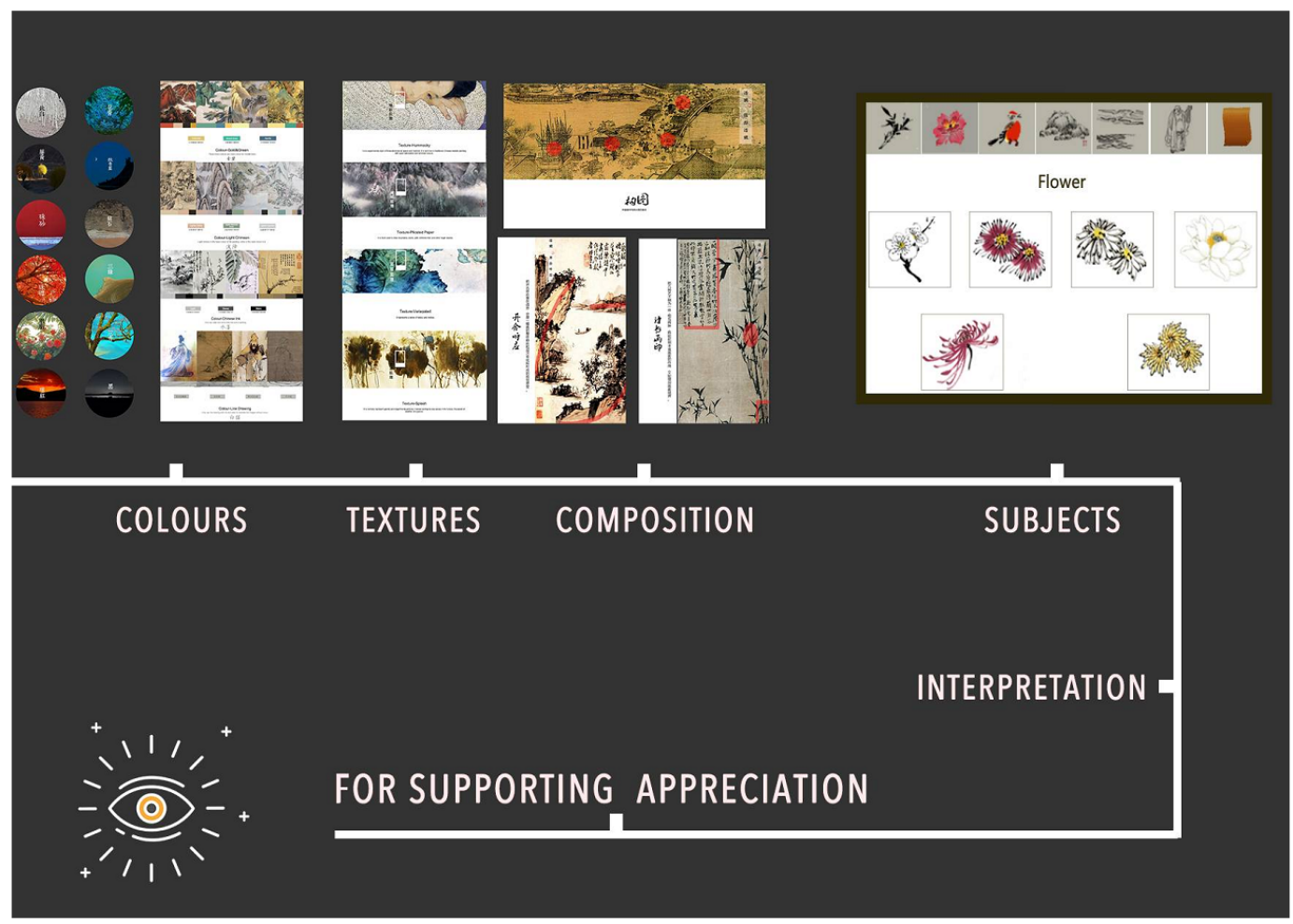

Figure 6. Interpretations of Chinese painting elements.

\subsection{Gestural Engagement and Multi-Touch in Chinese Painting}

Going further to engage traditional Chinese painting more in depth is very significant to non-Chinese audiences, and our participants gave their approval in the engagement of painting during the activities. Participants initially made a distinction between learning Chinese painting 
and appreciating/engaging Chinese painting. In this distinction they believed that: (i) Gestural engagement offered them more opportunities to appreciate the subtlety of the paintings. Through gestural movements (i.e., zoom in, zoom out, shifting, etc.), participants were able to also check details of the colours with traces, shades of ink, and the subtlety of textures in traditional Chinese painting. Figure 7 shows the gestures utilised numerous times by one of our participants. Participants are not only watching a piece of art being made or reading some related references, but also could gain a greater understanding of Chinese painting; (ii) By comparing how to learn to draw Chinese painting, participants were more inclined to use multi-touch tools to engage in painting instead of using a traditional ink brush. They believed that their main purpose was to appreciate the traditional features and culture in Chinese painting, rather than actually learning the skills. Sometimes, the technical skills of an ink-brush greatly reduced the participation and also the effort made by participants. During deployment, participants stated that archival elements with gestural operation sharply increased the integrity of the appreciation in Chinese painting, as well as offered participants a general understanding of the varying dimensions in traditional Chinese painting; (iii) It is noteworthy that the specific multi-touch gesture design was highlighted and discussed by participants on numerous occasions. What our participants did express was a desire for multiple users to use the graphic elements and to have the ability to interact with each other through this approach. Thus, the interoperability and compatibility of multi-gestural operations could be another design area for further development.

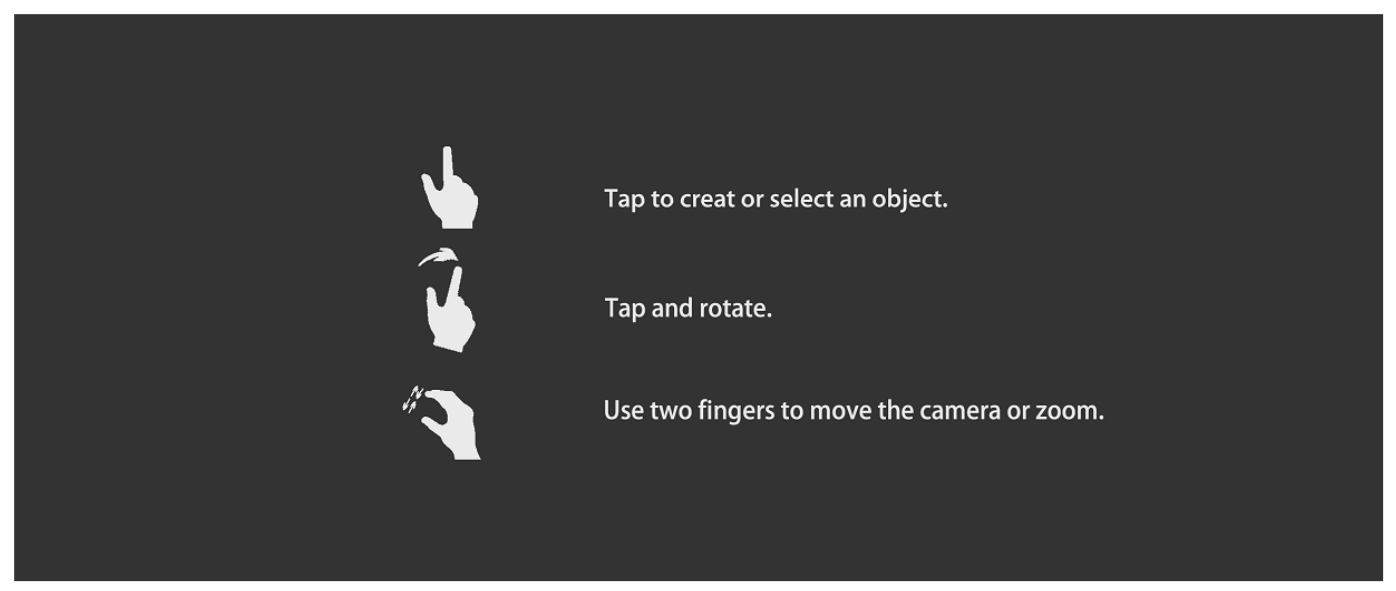

Figure 7. Three main gestures that mentioned by participants.

\subsection{Artworks Based on Mobile-Access}

Another area of the design insights is how to support audiences in achieving self-expression and collaborative engagement in Chinese painting. Based on the discussion of the two previous sections that integrated the elements archive and gestural operations, our participants expressed their demands through the application we designed. They preferred to utilise elements to express their own understanding and emotions within Chinese painting, as well as having an interaction with other participants to communicate their understanding with each other.

\subsubsection{Self-Expression}

After the participants had a basic understanding of the Chinese painting elements, engaging these participants in creating artwork could potentially support them to have an in-depth appreciation from their own expression and artistic collaborations with each other. We noticed that, when the participants expressed creating artwork with the application, the process of their appreciation became more active. Browsing the elements archive and checking the subtlety of the different elements formed an approach in supporting self-expression of participants, and rather than another appreciation approach we offered, participants checked websites or annotations passively and randomly. Meanwhile, participants wanted 
to express their own stories and emotions within the artwork. It needs to be emphasised that we created artwork rather than drawing paintings because the form of engagement was not focused solely on the painting skills themselves. Through communication with other participants, the aesthetic appreciation and stories of paintings are expected to be achieved more by participants.

\subsubsection{Collaborative Appreciation}

Different groups of participants (i.e., family members, university students, and amateurs) expressed their thoughts about collaborative interactions based on mobile equipment. Multiple user interactions that are not limited by specific occasions were requested by participants. For instance, participants could have a real-time interaction with other participants (who are in a different location) to use elements in creating an artwork based on their interaction and communication, no matter where they are. By using graphic elements from the archives in collaborative artwork, aesthetic appreciation and understanding will be discussed by the participants. The approaches of appreciation are not only limited by the personal reading material or knowledge teaching, so participants are highly expected to use the process of flexible collaborative appreciation. Meanwhile, the sharing of the collaborative appreciation has also been discussed, and sharing their artwork to engage with more participants within the already-existing engagement, could be another continuing method of appreciation.

\section{Conclusions}

We report field studies of a prototype interactive tablet application for the cross-cultural appreciation of Chinese painting. To support this, we conducted a cultural appreciation study with non-Chinese and Chinese participants to contrast the cultural differences that would explore the specific engagement approach for non-Chinese participants. Based on this, we designed an application targeted towards non-Chinese participants to explore interactive technology to support the appreciation and engagement of traditional Chinese painting. Our aim through this study was to offer valuable transferrable insights into the design of interactive technology that helps support the appreciation of traditional art. We raised a whole engagement approach and a series of interactive design suggestions for the support of cross-cultural appreciation during the development of the mobile application, focusing on the use of the elemental archive to support aesthetic appreciation; gestural/multi-touch for engagements; and the self-expression and collaborative appreciation through mobile-access. By addressing these design suggestions, we have arguably foregrounded feasibility and transferability of cross-cultural artistic appreciation and engagement, supported by digital interactive technology itself.

Acknowledgments: We would like to thank all participants who took part in our study.

Author Contributions: Shichao Zhao contributed to the design and planning of the study, the running of the interviews and workshops, analysis of the data, and the writing and revision of the manuscript. David Kirk contributed to the data analysis, and the writing and revision of the manuscript. Simon Bowen contributed to the data analysis, and the writing and revision of the manuscript. Peter Wright contributed to the data analysis, and the writing and revision of the manuscript.

Conflicts of Interest: The authors declare no conflict of interest.

\section{References}

1. Beardsley, M.C. The aesthetic point of view. In the Aesthetic Point of View: Selected Essays; Cornell University Press: Ithaca, NY, USA; London, UK, 1982; pp. 15-34.

2. Csikszentmihalyi, M.; Robinson, R. The Art of Seeing: An Interpretation of the Aesthetic Encounter; Getty Publications: Los Angeles, CA, USA, 1990; pp. 20-22.

3. Ryokai, K.; Misra, N.; Hara, Y. Artistic distance: Body movements as launching points for art inquiry. In Proceedings of the 33rd Annual ACM Conference Extended Abstracts on Human Factors in Computing Systems, Seoul, Korea, 18-23 April 2015; pp. 679-686.

4. Candy, L.; Ferguson, S. Interactive Experience in the Digital Age: Evaluating New Art Practice; Springer Series on Cultural Computing; Springer: London, UK, 2014. 
5. Huang, Y. Creation methodology of interactive art installation based on philosophy-understanding projection: Recreation of traditional Chinese painting. In Proceedings of the 2015 Virtual Reality International Conference, Laval, France, 8-10 April 2015; p. 7.

6. Kortbek, K.; Grønbæk, K. Communicating art through interactive technology: New approaches for interaction design in art museums. In Proceedings of the 5th Nordic Conference on Human-Computer Interaction: Building Bridges, Lund, Sweden, 20-22 October 2008; pp. 229-238.

7. Champion, E. Cross-cultural Learning, Heritage, and Digital Games. In Reorientation: Trans-Cultural. Trans-Cultural, Trans-Lingual, Transmedia Studies in Narrative, Language, Identity and Knowledge; Hartley, J., Qu, W., Eds.; Fudan University Press: Shanghai, China, 2016; pp. 218-233.

8. Pujol, L.; Champion, E. Evaluating presence in cultural heritage projects. Int. J. Heritage Stud. 2012, 18, 83-102. [CrossRef]

9. Creswell, J.W. Research Design: Qualitative, Quantitative and Mixed Methods Approaches; Sage: London, UK, 2002.

10. Glaser, B.G.; Strauss, A.L.; Strutzel, E. The discovery of grounded theory: Strategies for qualitative research. Nurs. Res. 1968, 17, 364. [CrossRef]

11. Wright, P.; McCarthy, J. Experience-Centred Design: Designers, Users, and Communities in Dialogue; Morgan and Claypool Publishers: San Rafael, CA, USA, 2010.

12. Lin, J.; Chen, Y.; Ko, J.; Kao, H.; Chen, W.; Tsai, T.; Hsu, S.; Hung, Y. I-m-Tube: An interactive multi-resolution tubular display. In Proceedings of the 17th ACM International Conference on Multimedia, Beijing, China, 19-24 October 2009; pp. 253-260.

13. Ma, W.; Kim, H.K.; Wang, Y.; Oh, W.G. Binocular stereopsis of traditional Chinese paintings. In Proceedings of the 10th International Conference on Virtual Reality Continuum and Its Application in Industry, Hong Kong, China, 11-12 December 2011; pp. 411-414.

14. Hsieh, C.; Hung, Y.; Ben-Ezra, M.; Hsieh, H. Viewing Chinese art on an interactive tabletop. IEEE Comput. Graph. Appl. 2013, 33, 16-21. [CrossRef] [PubMed]

15. Alexander, J.; Barton, J.; Goeser, C. Transforming the art museum experience: Gallery one. In Proceedings of the Museums and the Web, Portland, OR, USA, 17-20 April 2013; Proctor, N., Cherry, R., Eds.; 2013. Available online: https:/ / mw2013.museumsandtheweb.com/paper/transforming-the-art-museumexperience-gallery-one-2 (accessed on 25 June 2017).

16. Lang, D.; Findlater, L.; Shaver, M. CoolPaint: Direct interaction painting. In Proceedings of the 16th Annual ACM Symposium on User Interface Software and Technology, Vancouver, BC, Canada, 2-5 November 2003.

17. Otsuki, M.; Sugihara, K.; Kimura, A.; Shibata, F.; Tamura, H. MAI painting brush: An interactive device that realizes the feeling of real painting. In Proceedings of the 23nd Annual ACM Symposium on User Interface Software and Technology (UIST' 10), New York, NY, USA, 3-6 October 2010; pp. 97-100.

18. Vandoren, P.; Laerhoven, T.; Claesen, L.; Taelman, J.; Raymaekers, C.; Reeth, F. IntuPaint: Bridging the gap between physical and digital painting. In Proceedings of the 3rd IEEE International Workshop on Horizontal Interactive Human Computer System (TABLETOP' 08), Amsterdam, The Netherlands, 1-3 October 2008.

19. Zhang, J.; Lin, H.; Yu, J. A novel method for vectorizing historical documents of Chinese calligraphy. In Proceedings of the 10th IEEE International Conference on Computer Aided Design and Computer Graphics, Beijing, China, 15-18 October 2007; pp. 219-224.

20. Park, J. Digital canvas: A projection-space interaction tool. In Proceedings of the First International Conference on Technologies for E-Learning and Digital Entertainment (Edutainment' 06), Hangzhou, China, 16-19 April 2006; pp. 1171-1179.

21. Vandoren, P.; Claesen, L.; Laerhoven, T.; Taelman, J.; Raymaekers, C.; Flerackers, E.; Reeth, F. FluidPaint: An interactive digital painting system using real wet brushes. In Proceedings of the ACM International Conference on Interactive Tabletops and Surfaces, Banff, AB, Canada, 23-25 November 2009; pp. 53-56.

22. Huang, M.; Tang, W.; Lo, K.; Lau, C.; Ngai, G.; Chan, S. MelodicBrush: A novel system for cross-modal digital art creation linking calligraphy and music. In Proceedings of the Designing Interactive Systems Conference, Newcastle Upon Tyne, UK, 11-15 June 2012; pp. 418-427.

23. Jo, K. DrawSound: A drawing instrument for sound performance. In Proceedings of the 2nd International Conference on Tangible and Embedded Interaction, Bonn, Germany, 18-20 February 2008; pp. 59-62. 
24. Kang, L.; Gu, T.; Gay, G. Harmonic paper: Interactive music interface for drawing. In Proceedings of the Extended Abstracts on Human Factors in Computing System, Paris, France, 27 April-2 May 2013; pp. 763-768.

25. Yeom, J.; Lee, G. Designing a user interface for a painting application supporting real watercolor painting processes. In Proceedings of the 10th Asia Pacific Conference on Computer Human Interaction, Matsue-City, Shimane, Japan, 28-31 August 2012; pp. 219-226.

26. Huang, Y.; Lioret, A. Cerebral interaction and painting. In Proceedings of the SIGGRAPH Asia 2013 Art Gallery, Hong Kong, China, 19-22 November 2013; p. 21.

27. Zollinger, H. Color: A Multidisciplinary Approach; Wiley-VCH: New York, NY, USA, 1999; pp. $45-98$.

28. Lin, S. Conception of Color; SanMinBook Co., Ltd.: Taipei, Taiwan, 2000; pp. 133-136.

29. Yu, A. The Study of Colors in Traditional Chinese Painting; Beijing Joint Publishing Corporation: Beijing, China, 2013.

30. Baranauskas, C.; Palanque, P.; Abascal, J.; Diniz, S.; Barbosa, J. An eye tracking study of how pictures influence online reading. In Proceedings of the Human-Computer Interaction-INTERACT, Rio de Janeiro, Brazil, 10-14 September 2007; Volume 4667, pp. 456-460.

31. Chen, J.; Huang, Y. Research Report of Colour Preference; National Yunlin University of Science and Technology: Douliu, Taiwan, 1996; Volume 5, pp. 95-105.

32. Saito, M. A comparative study of colour preferences in Japan, China and Indonesia, with emphasis on the preference for white. Percept. Motor Skill 1996, 83, 115-128. [CrossRef] [PubMed]

33. Camgöz, N.; Yener, C.; Güvenç, D. Effects of hue, saturation, and brightness on preference. Color Res. Appl. 2002, 27, 199-207. [CrossRef]

34. Wang, X. Tradtional Chinese Painting Drawing Skills-Photoshop; Tsinghua University Press: Beijing, China, 2010.

35. Braun, V.; Clarke, V. Using thematic analysis in psychology. Qual. Res. Psychol. 2006, 3, 77-101. [CrossRef]

36. Chen, J.; Du, Y.; Li, H. Feature extraction and classification of Chinese painting. Comput. Eng. Appl. 2008, 44, 166-169.

37. Ji, Y. The Combination of Art and Technology in Interaction Design; EDN: Shanghai, China, 2013; pp. $18-21$.

38. Zhang, L. Discussion of emotional imagery expression of colors in Chinese painting. Coll. Henan Art 2012, 1, 36-39.

(c) 2018 by the authors. Licensee MDPI, Basel, Switzerland. This article is an open access article distributed under the terms and conditions of the Creative Commons Attribution (CC BY) license (http://creativecommons.org/licenses/by/4.0/). 